\title{
Relation between substorm characteristics and rapid temporal variations of the ground magnetic field
}

\author{
A. Viljanen ${ }^{1}$, E. I. Tanskanen ${ }^{1,2}$, and A. Pulkkinen ${ }^{2}$ \\ ${ }^{1}$ Finnish Meteorological Institute, Space Research Unit, P.O.B. 503, FI-00101 Helsinki, Finland \\ ${ }^{2}$ NASA/Goddard Space Flight Center, Code 612.2, Greenbelt, MD 20771, USA
}

\begin{abstract}
Auroral substorms are one of the major causes of large geomagnetically induced currents (GIC) in technological systems. This study deals with different phases of the auroral substorm concerning their severity from the GIC viewpoint. Our database consists of 833 substorms observed by the IMAGE magnetometer network in 1997 (around sunspot minimum) and 1999 (rising phase of the sunspot cycle), divided into two classes according to the $D_{s t}$ index: non-storm $\left(D_{s t}>-40 \mathrm{nT}, 696\right.$ events $)$ and storm-time ones ( $D_{s t}<-40 \mathrm{nT}, 137$ events). The key quantity concerning GIC is the time derivative of the horizontal magnetic field vector $(d \boldsymbol{H} / d t)$ whose largest values during substorms occur most probably at about $5 \mathrm{~min}$ after the onset at stations with CGM latitude less than $72 \mathrm{deg}$. When looking at the median time of the occurrence of the maximum $d \boldsymbol{H} / d t$ after the expansion onset, it increases as a function of latitude from about $15 \mathrm{~min}$ at $\mathrm{CGM}$ lat=56 deg to about $45 \mathrm{~min}$ at CGM lat $=75 \mathrm{deg}$ for non-storm substorms. For storm-time events, these times are about $5 \mathrm{~min}$ longer. Based on calculated ionospheric equivalent currents, large $d \boldsymbol{H} / d t$ occur mostly during the substorm onset when the amplitude of the westward electrojet increases rapidly.
\end{abstract}

Keywords. Geomagnetism and paleomagnetism (Rapid time variations) - Ionosphere (Auroral ionosphere) - Magnetospheric physics (Storms and substorms)

\section{Introduction}

Associated with geomagnetic variations, geomagnetically induced currents (GIC) flow in technological conductor systems such as power grids and pipelines (e.g. Boteler et al., 1998; Kappenman, 1996, 2005; Molinski, 2002). The basic principle follows from Faraday's law linking temporal

Correspondence to: A. Viljanen

(ari.viljanen@fmi.fi) changes of the magnetic flux to the electromotive force. So a geoelectric field is always associated with a temporally varying geomagnetic field. This means that the time derivative of the ground magnetic field $(d \boldsymbol{B} / d t)$ characterizes GIC activity. More explicitly, the horizontal component of the vector $(d \boldsymbol{H} / d t)$ is the relevant quantity (Viljanen et al., 2001). Calculation of GIC is practical in two independent steps: 1) determine the (horizontal) electric field at the earth's surface, 2) determine induced currents driven by this electric field in a specified conductor system. One technique used in postanalysis of GIC events is presented by Viljanen et al. (2004).

The ability to derive GIC statistics based on magnetic recordings can help utilities to estimate which parts of a conductor system might be susceptible to GIC effects. However, this does not help in preventing immediate damages, which may develop in a few minutes during magnetic disturbances. The best-known cases concerning power system failures are the Québec blackout in Canada in March 1989 (Bolduc, 2002), and the Malmö blackout in Sweden in October 2003 (Pulkkinen et al., 2005; Rosenqvist et al., 2005).

The present solar wind observations at the $\mathrm{L} 1$ point allow for a 30-60 min warning time in practice. It would be ideal if GIC forecasts could be provided at least one hour prior to anticipated large events, because certain system operating changes can take 1-2 h to complete (Molinski, 2002).

Exact local warnings would require that $d \boldsymbol{B} / d t$ could be forecasted as a time series close to the sites where GIC are of interest. The surface grid could be quite sparse (of a 100$\mathrm{km}$ scale), but it would be critical to predict $d \boldsymbol{B} / d t$ accurately, especially concerning the largest values. Weigel et al. (2003) has attempted to forecast the 30-min average absolute magnitude of $d \boldsymbol{B} / d t$ directly from solar wind observations. The highest prediction efficiencies achieved are about 0.7 . The success of the prediction is highly dependent on the spatial location and on the local time. Wintoft (2005) showed that the 10-min log-root-mean-square of $d X / d t$ and $d Y / d t$

Published by Copernicus GmbH on behalf of the European Geosciences Union. 


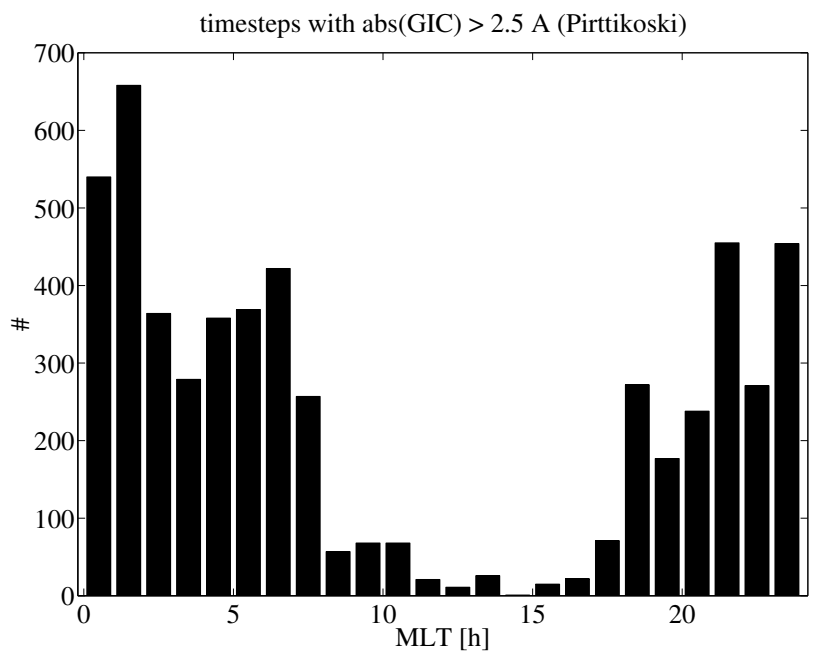

Fig. 1. Diurnal variation of the number of observations in 19992000 when the absolute value of GIC (10-s data) exceeded $2.5 \mathrm{~A}$ at the Pirttikoski 400-kV transformer in northern Finland. The corrected geomagnetic (CGM) latitude of this site is about $63 \mathrm{deg} \mathrm{N}$. The figure is taken from Viljanen et al. (2001).

at two subauroral locations may be predicted up to $30 \mathrm{~min}$ in advance with a linear correlation coefficient close to 0.8 .

The main difficulty in GIC forecasting is obviously the large variability of the length scales of ionospheric current systems relevant to GIC (Pulkkinen et al., 2003a,b). Viljanen et al. (1999) listed some event types in their Table 1, during which large GIC may occur. A manual rough classification of one-year GIC data indicated that rapid enhancements of large-scale auroral electrojets, substorm onsets, pulsations, and sudden impulses have caused significant GIC. The diurnal occurrence of large GIC values at one site in northern Finland is shown in Fig. 1. There is a clear maximum around the magnetic midnight corresponding most probably to substorm activity. This gives the motivation for this paper to investigate auroral substorms in detail from the GIC viewpoint. Since GIC measurements are not available as extensively as magnetic recordings, we use the latter ones.

An enhanced geomagnetic activity is obviously a precondition for the occurrence of large GIC. However, a large magnetic variation field $\boldsymbol{B}$ itself does not necessarily imply that $d \boldsymbol{B} / d t$ is also large, and vice versa. As shown by Viljanen et al. (2001), large $d \boldsymbol{B} / d t$ events are nearly always related to westward ionospheric currents. However, the directional distributions of the horizontal time derivative vector $(d \boldsymbol{H} / d t)$ are much more scattered than those of the simultaneous horizontal variation field vector $(\boldsymbol{H})$. This is possible only if there are rapidly changing ionospheric current systems of a length scale of $100 \mathrm{~km}$ or less embedded in a smooth background east-west flow. Pulkkinen et al. (2003a), Pulkkinen et al. (2003b) and Viljanen et al. (2004) have demonstrated this

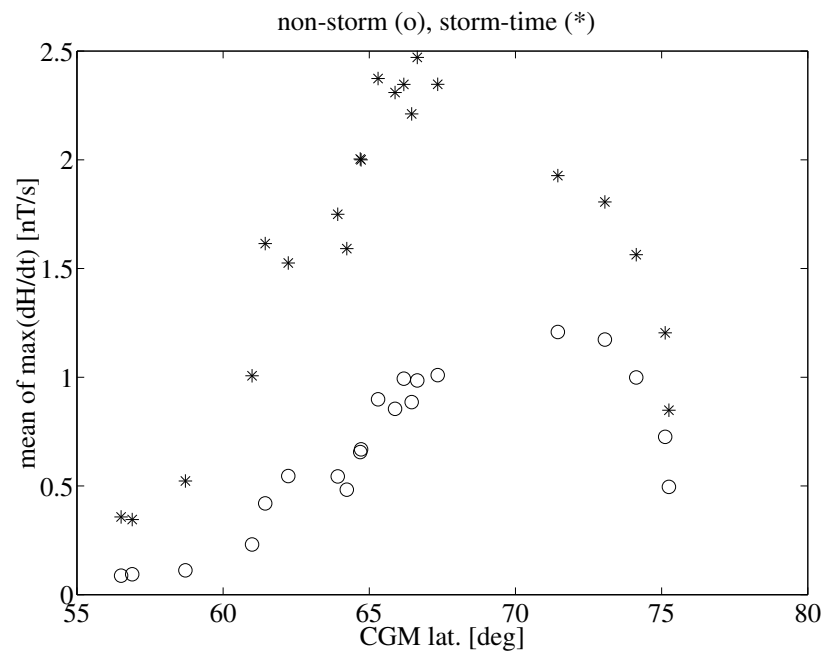

Fig. 2. Average of $\max (|d \boldsymbol{H} / d t|)$ during substorms as a function of the CGM latitude. Circles: non-storm substorms, asterisks: stormtime substorms.

with single events. It is obvious that GIC forecasting cannot be based on solar wind data only, but conditions in the magnetosphere and ionosphere are important too.

The main science question to which we are looking for an answer in this paper is how the GIC activity varies during different substorm phases. We start examining characteristics of $d \boldsymbol{H} / d t$ followed by a study of the temporal and spatial behaviour of ionospheric equivalent currents during substorms.

\section{Results}

\subsection{Data description}

Our database consists of 833 substorms in 1997 (around sunspot minimum) and 1999 (rising phase of the sunspot cycle 23) observed by the IMAGE magnetometer network in northern Europe (Tanskanen et al., 2002). IMAGE covers the magnetic latitudes where the electrojets related to substorms are flowing. We used the local variant of the AL index (IL index) to define a substorm. We looked for negative bay-type variations of IL (>100 nT) showing a clear onset when IL rapidly decreases at least $100 \mathrm{nT}$ within $10 \mathrm{~min}$. We considered the periods between 16:00 UT and 02:00 UT (about 18:00-04:00 MLT), when IL gives a good estimate of the global AL. The exact timing of the onset is somewhat subjective, so there is a couple of minutes inaccuracy. We divided the events into two classes, non-storm and stormtime ones, according to the $D_{s t}$ index. The value of $D_{s t}$ is smaller than $-40 \mathrm{nT}$ for storm-time substorms and larger than $-40 \mathrm{nT}$ for non-storm ones, respectively (Kallio et al., 2000). In this dataset, there are 696 non-storm and 137 storm-time substorms (from the original list of Tanskanen et al. (2002), 


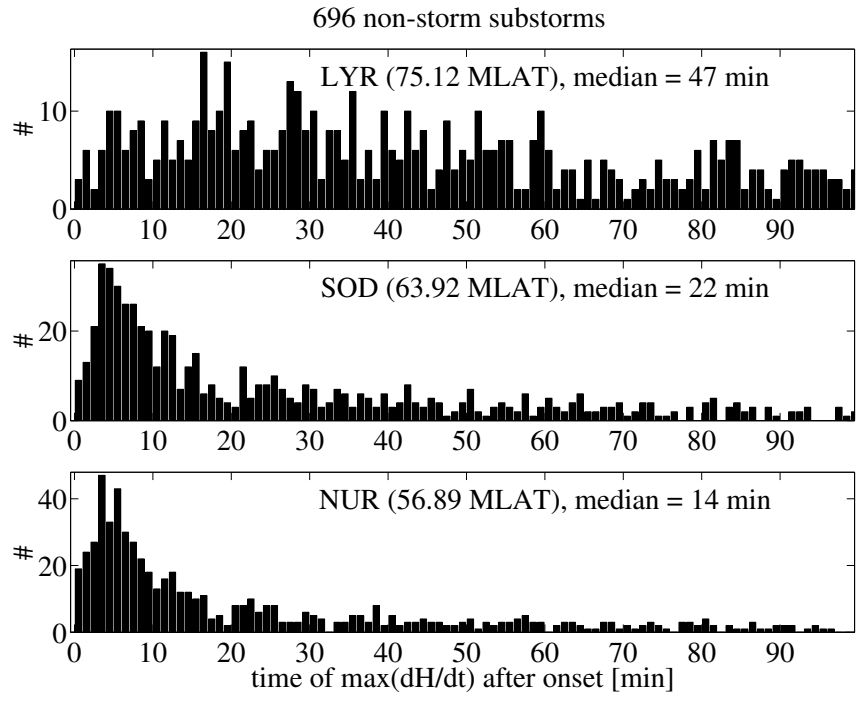

137 storm-time substorms
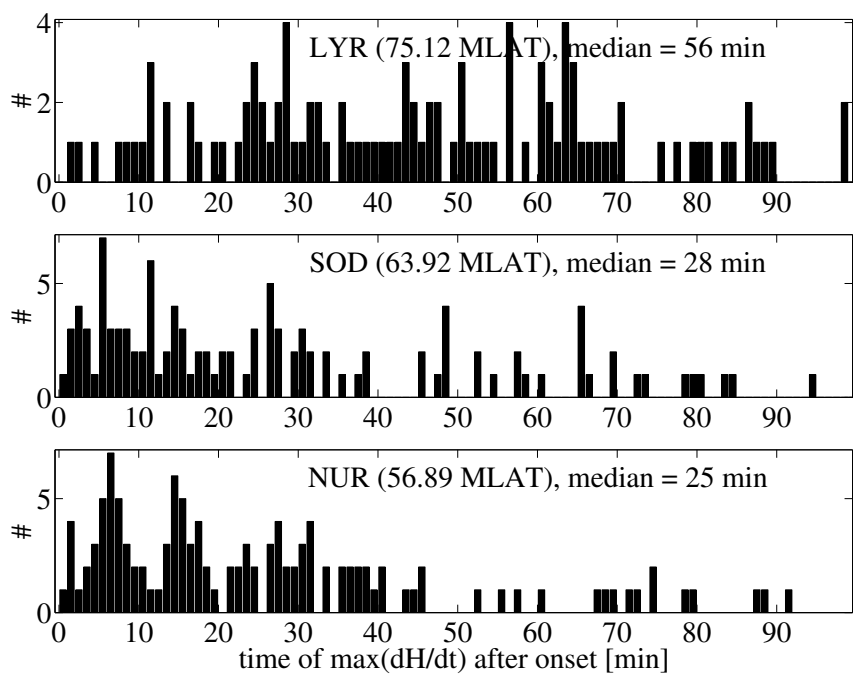

Fig. 3. Time of $\max (|d \boldsymbol{H} / d t|)$ after the substorm onset at three IMAGE sites: LYR, SOD, NUR. Upper panel: non-storm substorms, lower panel: storm-time substorms.

6 events were omitted). The time resolution of the magnetic data is $1 \mathrm{~min}$.

We use the magnetic variation field from which the quiettime baseline is subtracted. The baselines have been selected visually for each day separately. The time derivative of the field is the difference between two successive values divided by the sampling interval, and it is independent of the baseline. One minute is still a sufficiently short time step to reveal characteristic features of $d \boldsymbol{H} / d t$.

\subsection{Characteristics of $d \boldsymbol{H} / d t$}

The average of the maximum $|d \boldsymbol{H} / d t|$ at each IMAGE site is shown in Fig. 2. During storm-time substorms,
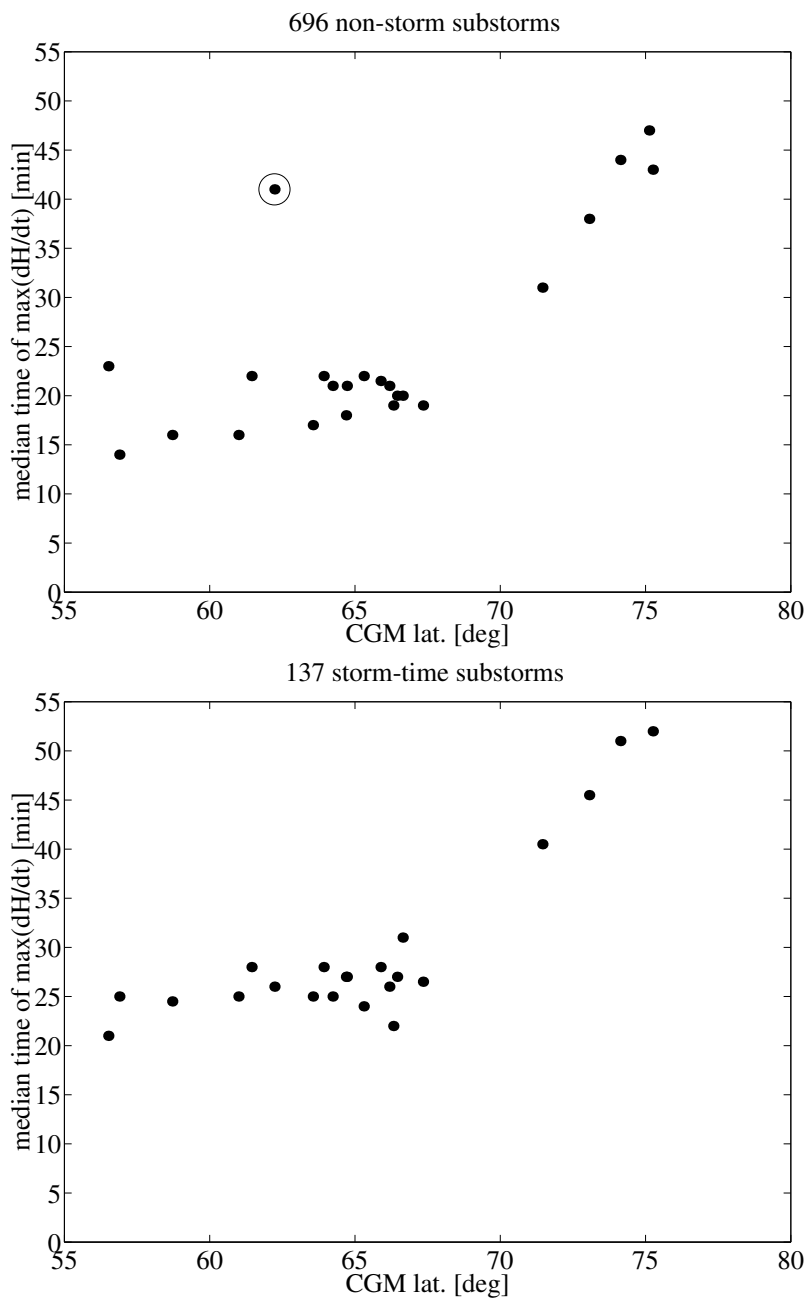

Fig. 4. The median time of $\max (|d \boldsymbol{H} / d t|)$ after the onset as a function of the CGM latitude. Upper panel: non-storm substorms, lower panel: storm-time substorms. A clear outlier is marked by a circle.

$\max (|d \boldsymbol{H} / d t|)$ is approximately twice the value of non-storm substorms at all latitudes. The site of maximum $|d \boldsymbol{H} / d t|$ as a function of latitude is about $5 \mathrm{deg}$ more southward for stormtime than for non-storm substorms. The gap between the latitudes 67 and 71 is due to the Arctic Ocean between the mainland and Bear Island.

The occurrence time of $\max (|d \boldsymbol{H} / d t|)$ after the substorm onset at each available magnetometer site gives an overview of the GIC activity during substorms. Examples of occurrence distributions at single sites are shown in Fig. 3. For non-storm substorms, distributions are sharply peaked at lower latitudes (NUR, SOD) as indicated by the maxima at about $5 \mathrm{~min}$ after the onset. However, there is still a clear tail in the distribution, which is typical for complex multiscale systems (Sornette, 2004). Distributions of $\max (|d \boldsymbol{H} / d t|)$ are scattered for storm-time substorms at all sites, which may 

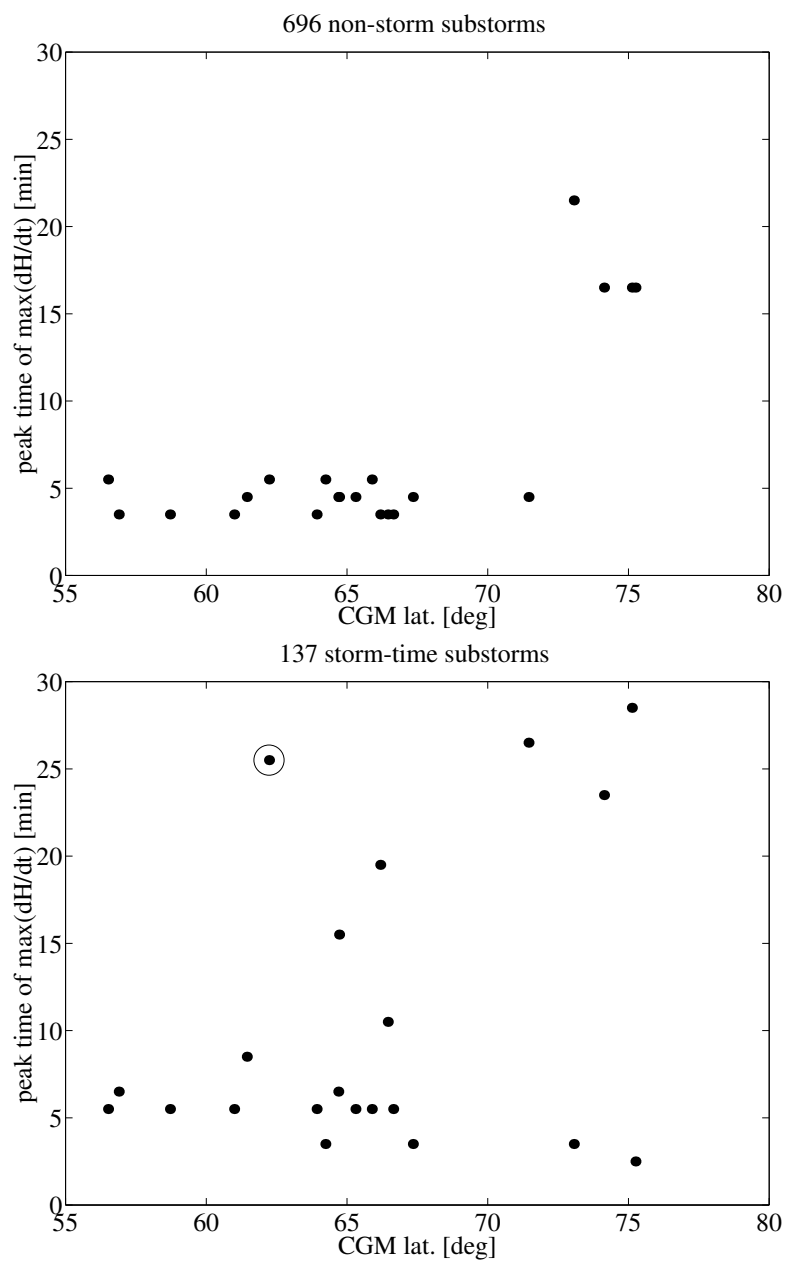

Fig. 5. The most probable time of $\max (|d \boldsymbol{H} / d t|)$ after the onset as a function of the CGM latitude. Upper panel: non-storm substorms, lower panel: storm-time substorms. A clear outlier is marked by a circle.

also be due to the relatively small number of data. For a more quantitative comparison of the distributions, we performed a simple $\chi^{2}$ test (e.g. Press et al., 2002) between pairs of stations using time bins from 0 to $60 \mathrm{~min}$. We then obtained the following $\chi^{2}$ values with the corresponding probabilities in parantheses. Non-storm substorms yield NUR-SOD 47.3 (0.86), NUR-LYR 244 (0.00), SOD-LYR 187 (0.00), and storm-time cases yield NUR-SOD 36.2 (0.99), NURLYR 70.1 (0.15), SOD-LYR 74.9 (0.08). This means roughly that the distributions at LYR are really different from those at SOD and NUR, whereas SOD and NUR are quite similar to each other.

The median value of the occurrence time of $\max (|d \boldsymbol{H} / d t|)$ increases as a function of latitude (Fig. 4) for both nonstorm and storm-time cases. The Svalbard stations (CGM lat $>70$ ), including Bear Island, differ clearly from the continental sites. In the non-storm case the station at CGM lat 62.23

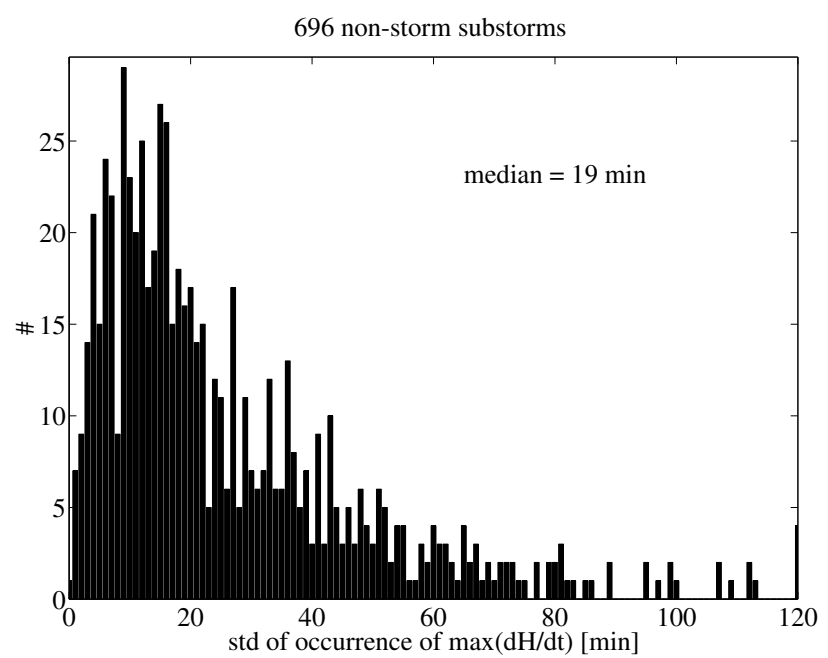

137 storm-time substorms

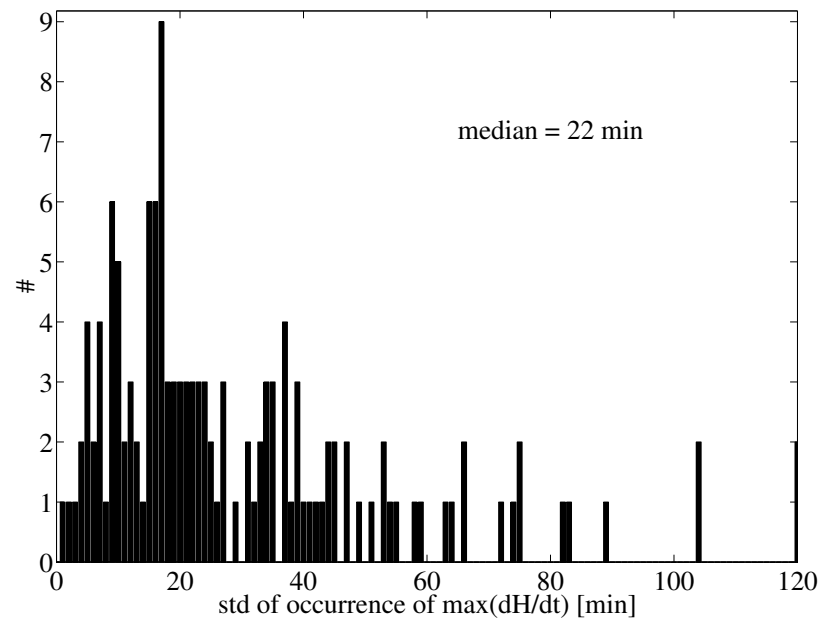

Fig. 6. Simultaneity of the occurrence of $\max (|d \boldsymbol{H} / d t|)$ after the onset at the IMAGE sites. The histogram presents the standard deviation of the maxima times. Events with data from at least 15 stations are taken into account. Upper panel: non-storm substorms, lower panel: storm-time substorms.

(Rørvik) is a clear outlier due to the small amount of available data.

When the most probable occurrence time of $\max (|d \boldsymbol{H} / d t|)$ after onset is considered (Fig. 5), the situation becomes more complex. For non-storm substorms, the four sites in Svalbard (CGM lat >73) and the continental sites and Bear Island (CGM lat $<71.5$. deg) still comprise two distinct sets. The Svalbard stations are mostly north of the auroral oval around the local midnight (cf. Kauristie, 1995), so they do not show typical substorm features. Storm-time events in turn have quite an irregular behaviour as a function of latitude, evidently due to the small number of datapoints. However, also for them, the most probable maximum time is about $5 \mathrm{~min}$ after the onset at lowest latitudes. 

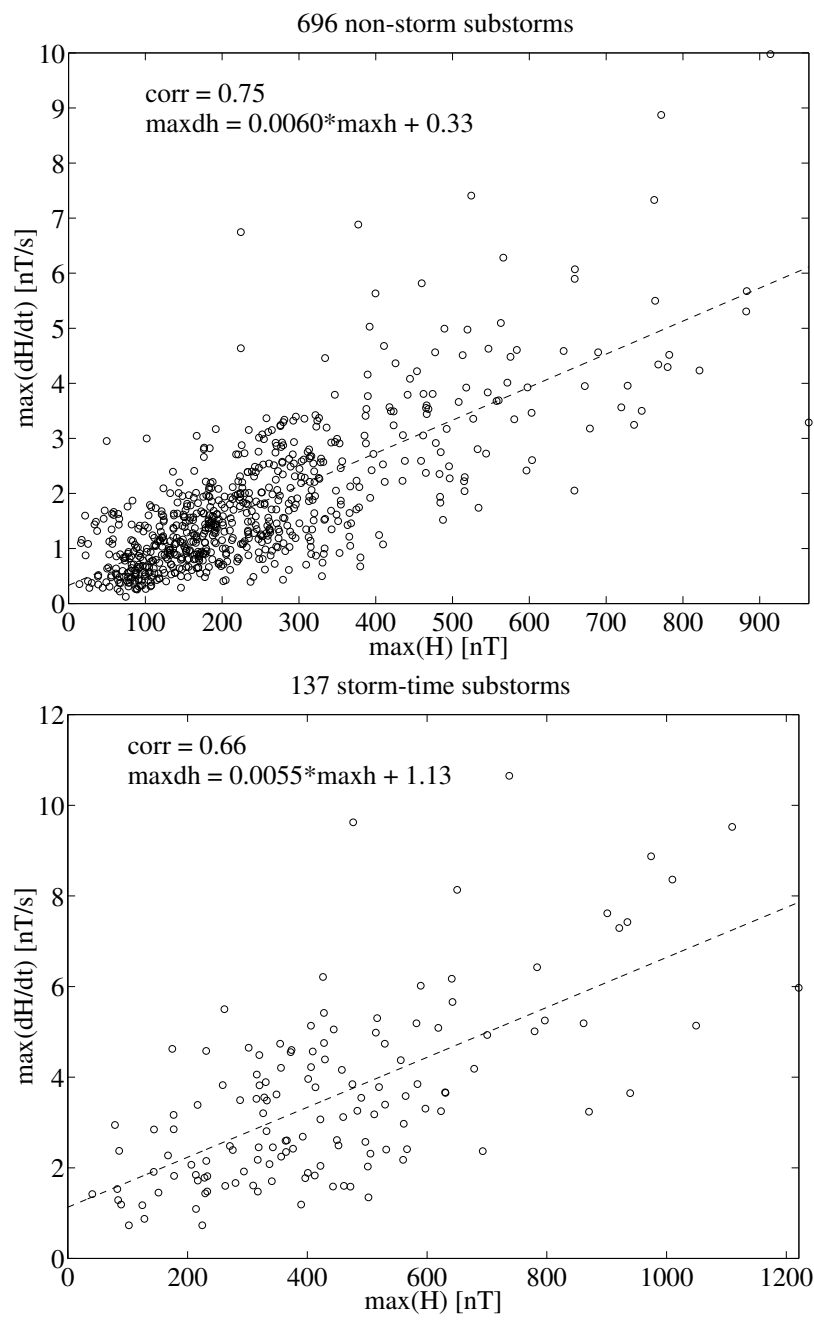

Fig. 7. The $\max (|d \boldsymbol{H} / d t|)$ versus $|\boldsymbol{H}|$ at the maximum times of $|d \boldsymbol{H} / d t|$ during non-storm (upper panel) and storm-time (lower panel) substorms. The dashed line is the linear regression curve.

The characteristics of a GIC event also depend on how simultaneously large $d \boldsymbol{H} / d t$ values occur. To quantify this, we have collected maximum times of $d \boldsymbol{H} / d t$ at all sites and calculated the standard deviation of them for each event. To have a good spatial coverage, we have only considered events with data available at least from 15 sites. A complete simultaneity would produce a zero standard deviation, but in practice there is much scattering with a clearly tailed distribution as shown in Fig. 6. The simultaneity does not directly give any information about GIC values at a specific site, since the geometry of the induced geoelectric field should also be known.

An interesting open question is how closely $|d \boldsymbol{H} / d t|$ is related to $|\boldsymbol{H}|$. Knowing the relationship may improve forecasting possibilities. There is already quite a good success in predicting $|\boldsymbol{H}|$ or indices based on it, like AE (Gleisner
696 non-storm substorms: $\max (\mathrm{dH} / \mathrm{dt})$

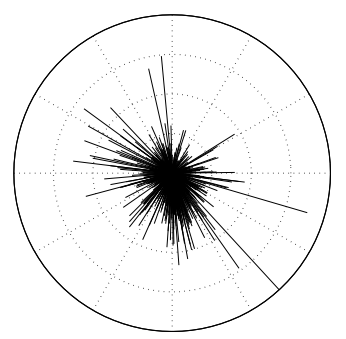

$\max =10.0 \mathrm{nT} / \mathrm{s}$

137 storm-time substorms: $\max (\mathrm{dH} / \mathrm{dt})$

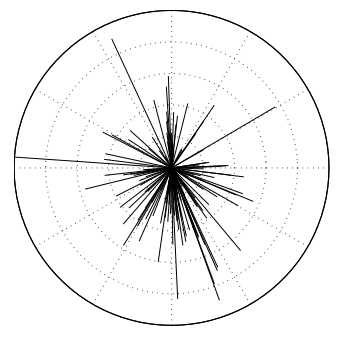

$\max =10.7 \mathrm{nT} / \mathrm{s}$

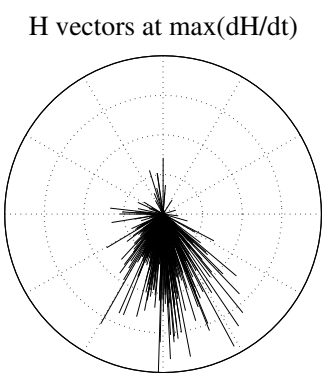

$\max =964 \mathrm{nT}$

$H$ vectors at $\max (\mathrm{dH} / \mathrm{dt})$

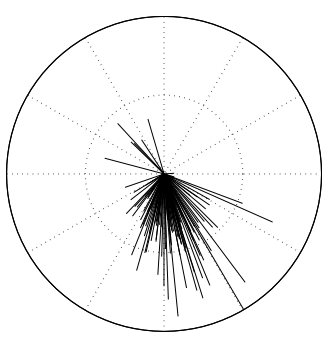

$\max =1221 \mathrm{nT}$

Fig. 8. Upper row: $\max (d \boldsymbol{H} / d t)$ vectors of non-storm substorms, and simultaneous $\boldsymbol{H}$ vectors at the maximum sites of $d \boldsymbol{H} / d t$. Lower row: the same for storm-time substorms. The north direction is up and the east direction to the right.

and Lundstedt, 1997). The relation between $\max (|\boldsymbol{H}|)$ and $\max (|d \boldsymbol{H} / d t|)$ for non-storm and storm-time substorms are shown in Fig. 7. These plots deal with the maximum value of $|d \boldsymbol{H} / d t|$ during each substorm with the simultaneous $|\boldsymbol{H}|$ at the maximum site of $|d \boldsymbol{H} / d t|$. There is quite a high correlation between $|\boldsymbol{H}|$ and $|d \boldsymbol{H} / d t|, 0.75$ for non-storm and 0.66 for storm-time substorms. However, for a given $|\boldsymbol{H}|$, the range of $|d \boldsymbol{H} / d t|$ values is roughly $\pm 2 \mathrm{nT} / \mathrm{s}$. The slope of the regression curve is nearly the same for both types of substorms. This indicates that the (substorm) mechanism behind the relation between $\boldsymbol{H}$ and $d \boldsymbol{H} / d t$ may be independent of storm conditions.

A clear majority of $\max (|d \boldsymbol{H} / d t|)$ is associated with a westward electrojet as shown in Fig. 8, since a southward variation field is due to a westward (equivalent) current flow in the ionosphere. The scattering of $d \boldsymbol{H} / d t$ means that rapid changes are not always due to an increase or decrease of the electrojet current, but they are often related to some smaller scale structures like vortices (Viljanen et al., 2001; Apatenkov et al., 2004).

The spatial occurrence of the maximum $d \boldsymbol{H} / d t$ is shown in Fig. 9. The average maximum site is more southward for storm-time substorms, and reflects the well-known shift of the auroral oval equatorwards during increased magnetic activity (e.g. Rostoker and Phan, 1986; Ahn et al., 2005). The same feature is obvious in Fig. 2. The dominance of some 

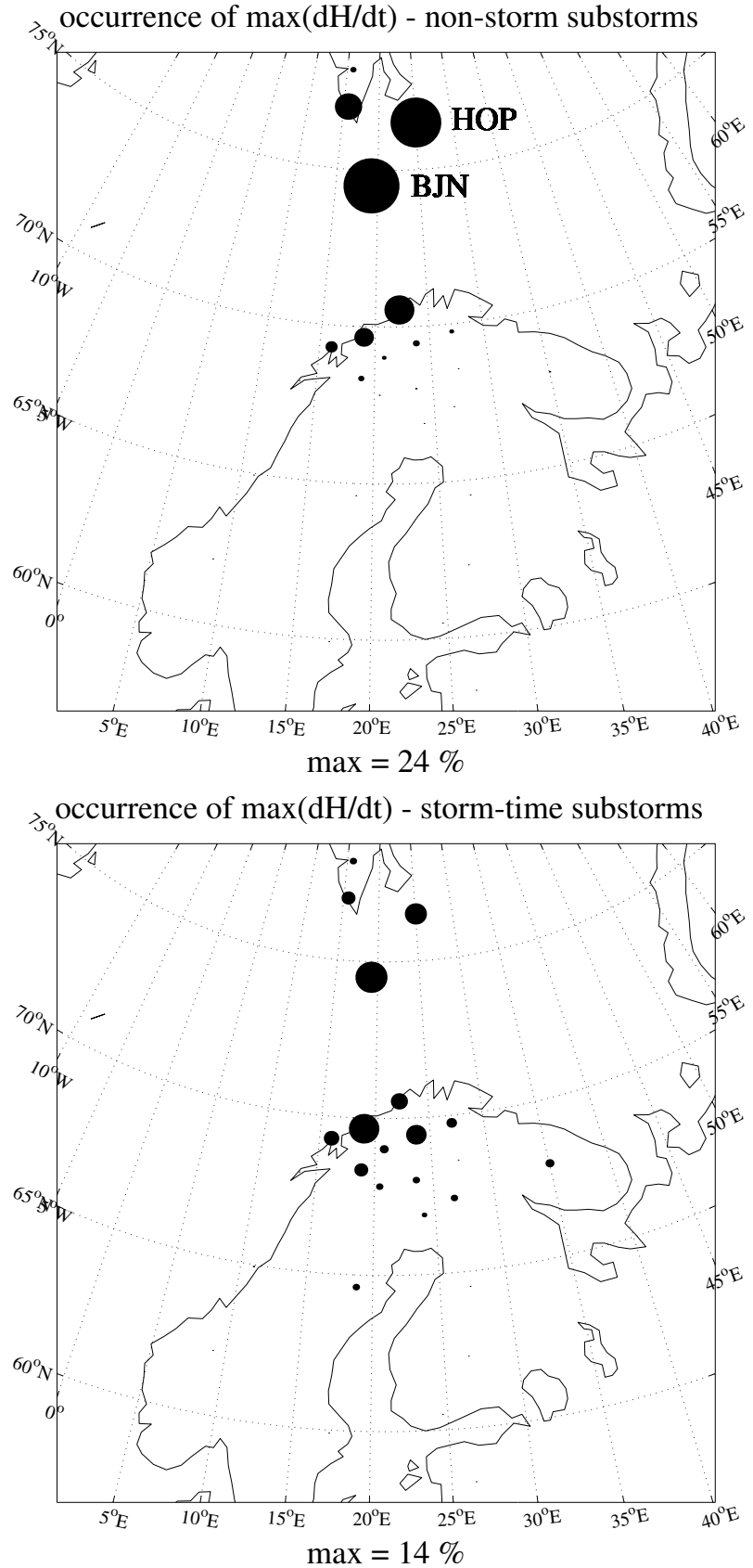

Fig. 9. Spatial occurrence distribution of the maximum site of $d \boldsymbol{H} / d t$ during non-storm (upper panel) substorms and storm-time substorms (lower panel).

sites is partly due to the local conductivity of the earth's upmost crust: the larger the conductivity the larger $d \boldsymbol{H} / d t$ can be at short periods with small skin depths (Viljanen et al., 2001). Especially, the high conductivity of the ocean water has an obvious effect at BJN and HOP stations. On the other hand, $\boldsymbol{H}$ is less sensitive to the local earth conditions, since its power spectrum has largest amplitudes at longer periods
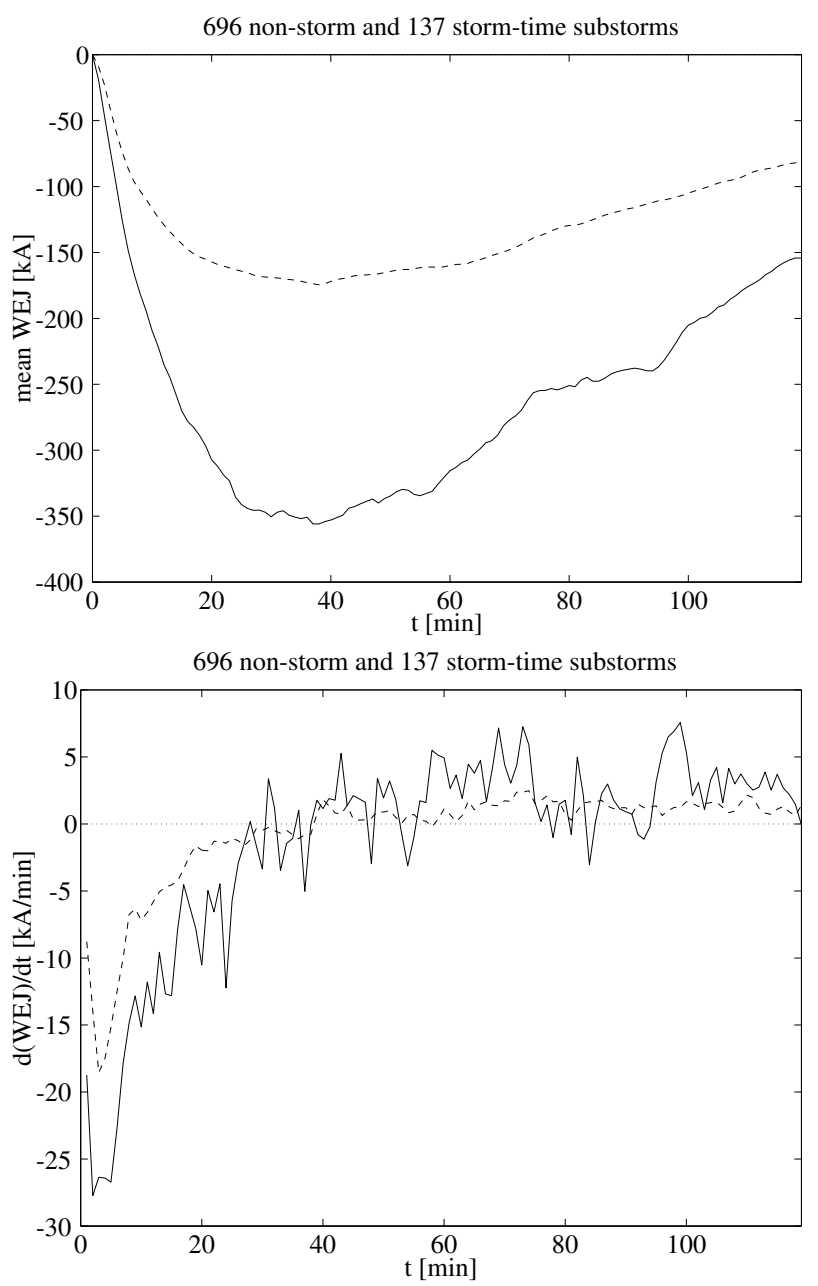

Fig. 10. Mean total westward electrojet (WEJ) and its time derivative during a non-storm (dashed line) and a storm-time substorm (uniform line). The origin of the time axis is the substorm onset, when the current is set to zero.

than the time derivative. Consequently, relevant skin depths are larger for $\boldsymbol{H}$ than for $d \boldsymbol{H} / d t$.

\subsection{Ionospheric equivalent currents}

Ionospheric equivalent currents have been calculated using the method of spherical elementary current systems (Amm, 1997; Pulkkinen et al., 2003a). The current density was determined along the geographic longitude $22.06 \mathrm{deg} \mathrm{E}$ from the latitude $59.02 \mathrm{~N}$ to $79.42 \mathrm{~N}$ with a 0.6 -deg spacing. Since we did not separate the ground magnetic field into external and internal parts, the equivalent current actually contains the induced currents in the earth. This overestimates the ionospheric contribution up to $40 \%$ at onset times close to the maximum current flow (Tanskanen et al., 2001). However, it is not a drawback from the viewpoint of the present study, 

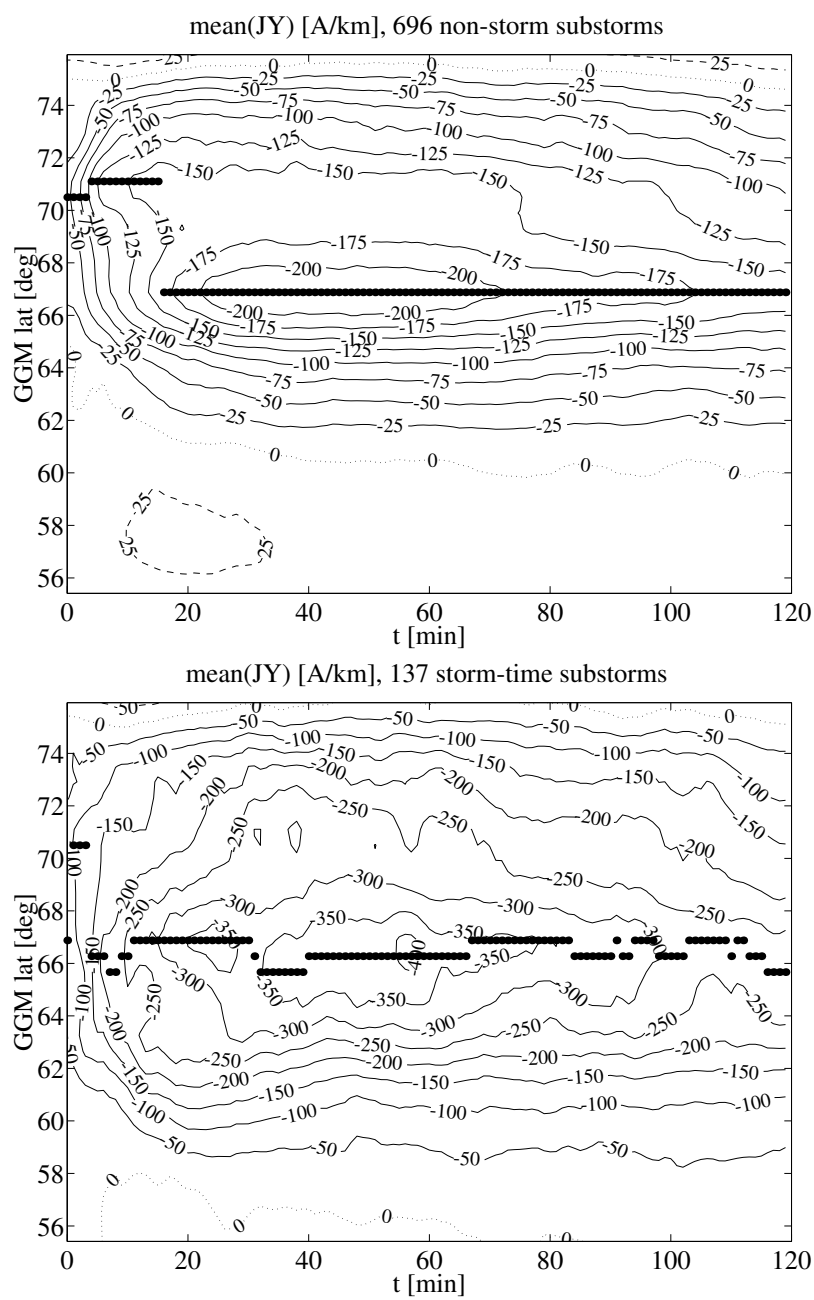

Fig. 11. Average eastward equivalent current density $(J Y)$ during $120 \mathrm{~min}$ after the onset at $t=0$. Negative values marked by negative contour lines mean a westward current. Dots show the location of the maximum westward current density. Upper panel: non-storm substorms, lower panel: storm-time substorms.

since GIC is related to the total $d \boldsymbol{H} / d t$ at the earth's surface.

The mean temporal behaviour of the total westward current is shown in Fig. 10. We set the total current to zero at the onset time, which does not affect the shape of the curves. The curves shown in the figures are the averages of all events. The time derivative was calculated from the average curve of the total current. As expected, the total current is more intense during storm-time events. The maximum of the derivative occurs a few minutes after the onset, which is consistent with Fig. 3 (stations NUR and SOD). Due to using the integrated total electrojet, the behaviour of the time derivative reflects mostly the large-scale characteristics of ionospheric currents.

The average equivalent ionospheric current density with respect to time and magnetic latitude is shown in Fig. 11.
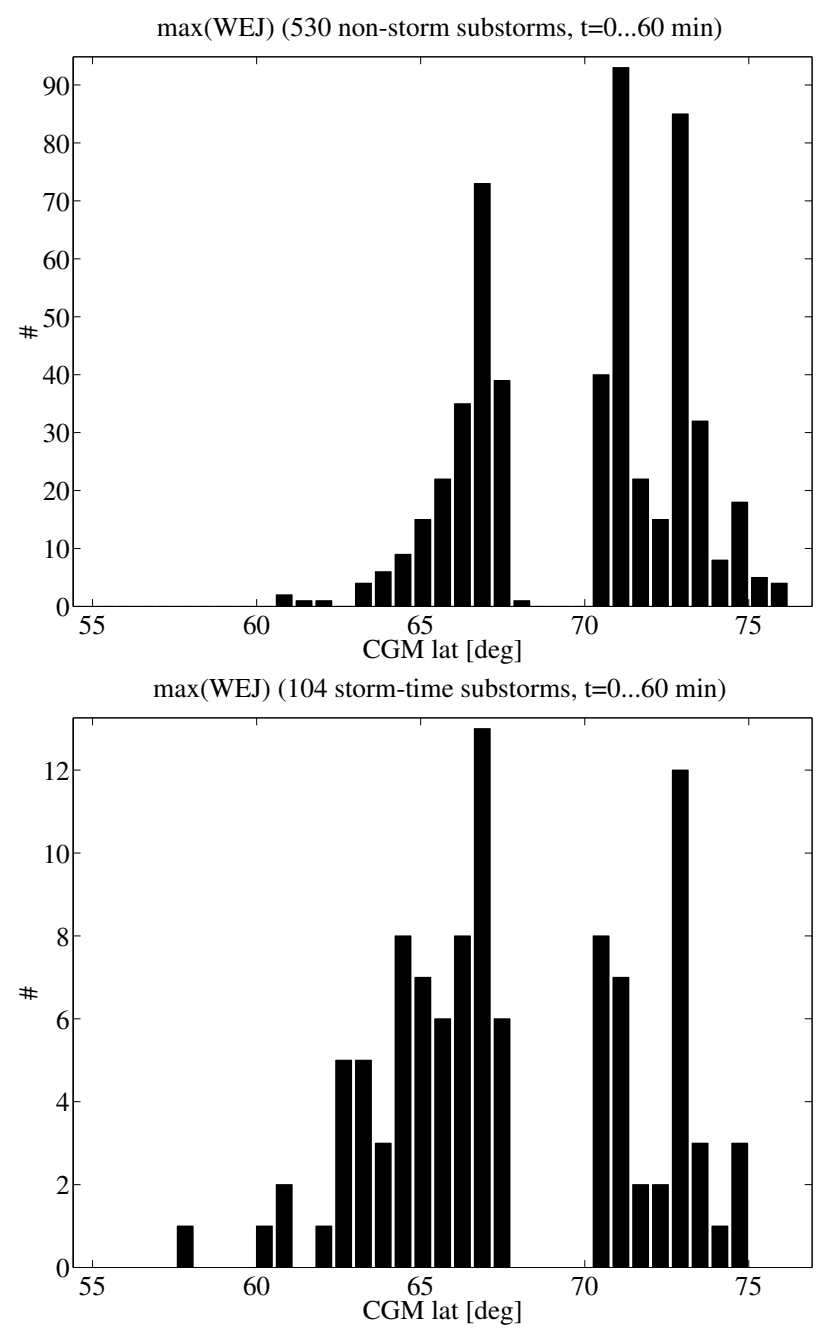

Fig. 12. Distribution of the latitude of the maximum of the westward equivalent current (WEJ) density at the moment of the maximum $d \boldsymbol{H} / d t$. Only timesteps within $60 \mathrm{~min}$ after the onset are taken into account.

The largest westward current takes place at about geomagnetic latitude 70.5, 1 deg south of Bear Island (BJN in Fig. 9). However, during storm-time substorms, the maximum moves in a few minutes $4 \mathrm{deg}$ southward above the continental coastline. The same happens for non-storm cases, but only after about $15 \mathrm{~min}$ after the onset. When analysing these features, we must note that there is a gap in IMAGE between latitudes 67.5 and 70.5 due to the Arctic Ocean. Furthermore, the network is sparse north of Bear Island too. The method to calculate equivalent currents does not tend to produce maxima of the current density in such gaps. It follows that the prominent asymmetry in the latitudinal distribution of the current density may not be true.

The latitude of the maximum westward current density at the moment of the maximum $d \boldsymbol{H} / d t$ is shown in Fig. 12. It 
is very rare that the maximum takes place at CGM latitudes lower than 60. This is in a good agreement with the results by Ahn et al. (2005). Again, the gap between latitudes 67.5 and 70.5 is an artifact due to the lack of stations. Finally, we determined the latitude of the maximum westward current density at the time of the largest $|d \boldsymbol{H} / d t|$ after onset. It is quite well linearly related to the latitude of $\max (|d \boldsymbol{H} / d t|)$ with the correlation coefficient of 0.78 .

\section{Conclusions}

The key quantity concerning geomagnetically induced currents is the time derivative of the horizontal magnetic field vector $(d \boldsymbol{H} / d t)$, which is available on wide ground networks with good spatial and temporal coverages. Based on the study of 833 substorms, the largest values of $d \boldsymbol{H} / d t$ occur most probably during the substorm expansion phase at about five min after the expansion onset at stations with CGM latitudes less than $72 \mathrm{deg}$. However, the occurrence distribution has a long tail extending to tens of minutes. When looking at the median time of the occurrence of the maximum $d \boldsymbol{H} / d t$ after the onset, it increases as a function of latitude from about $15 \mathrm{~min}$ at CGM lat $56 \mathrm{deg}$ to about $45 \mathrm{~min}$ at CGM lat $75 \mathrm{deg}$ for non-storm substorms.

The maximum $d \boldsymbol{H} / d t$ can have a large east-west component compared to the north-south one, whereas the simultaneous $\boldsymbol{H}$ is more concentrated into the southward direction. This indicates that in addition to the rapid change of the main electrojet, there are often ionospheric structures of smaller scales having a comparable effect on $d \boldsymbol{H} / d t$. In this sense, there seems to be no differences between non-storm and storm-time substorms except for in amplitudes, which are larger for the latter ones, and occur more southward. McPherron and Hsu (2002) found no qualitative distinction between the various classes of substorms, but they are likely to be caused by the same mechanism, and our results give some further support to this.

In this paper the relationship between the GIC activity, charaterised by the time derivative of the ground magnetic field, and substorm activity was examined for two years of which 1997 occurs around the sunspot minimum and 1999 at the rising phase of the sunspot cycle 23. A future study is needed to explore the GIC-substorm relationship in the descending sunspot cycle phase, when high speed streams are known to have a large effect on the substorm activity (Tanskanen et al., 2005).

Acknowledgements. IMAGE magnetometer data are collected as an international project headed by the Finnish Meteorological Institute. We thank Fingrid Oyj and Gasum Oy companies for their long-term interest in GIC studies in Finland. The work of E. I. Tanskanen was supported by the National Research Council in the US and Academy of Finland, and the work by A. Pulkkinen was performed when holding a National Research Council Associateship Award at NASA Goddard Space Flight Center.
Topical Editor I. A. Daglis thanks two referees for their help in evaluating this paper.

\section{References}

Ahn, B.-H., Chen, G. X., Sun, W., Gjerloev, J. W., Kamide, Y., Sigwarth, J. B., and Frank, L. A.: Equatorward expansion of the westward electrojet during magnetically disturbed periods, J. Geophys. Res., 110, doi:10.1029/2004JA010553, 2005.

Amm, O.: Ionospheric Elementary Current Systems in Spherical Coordinates and Their Application, J. Geomag. Geoelectr., 49, 947-955, 1997.

Apatenkov, S. V., Sergeev, V. A., Pirjola, R., and Viljanen, A.: Evaluation of the geometry of ionospheric current systems related to rapid geomagnetic variations, Ann. Geophys., 22, 63-72, 2004.

Bolduc, L.: GIC observations and studies in the Hydro-Quebec power system, J. Atmos. Sol.-Terr. Phys., 64, 1793-1802, 2002.

Boteler, D. H., Pirjola, R. J., and Nevanlinna, H.: The effects of geomagnetic disturbances on electrical systems at the earth's surface, Adv. Space Res., 22, 17-27, 1998.

Gleisner, H. and Lundstedt, H.: Response of the auroral electrojets to the solar wind modeled with neural networks, J. Geophys. Res., 102(A7), 14 269-14 270, 1997.

Kallio, E. I., Pulkkinen, T. I., Koskinen, H. E. J., Viljanen, A., Slavin, J. A., and Ogilvie, K.: Loading-Unloading Processes in the Nightside Ionosphere, Geophys. Res. Lett., 27, 1627-1630, 2000.

Kappenman, J. G.: Geomagnetic Storms and Their Impact on Power Systems, IEEE Power Engineering Review, May 1996, 5-8, 1996.

Kappenman, J. G.: An overview of the impulsive geomagnetic field disturbances and power grid impacts associated with the violent Sun-Earth connection events of 29-31 October 2003 and a comparative evaluation with other contemporary storms, Space Weather, 3, S08C01, doi:10.1029/2004SW000128, 2005.

Kauristie, K.: Statistical fits for auroral oval boundaries during the substorm sequence, J. Geophys. Res., 100(A11), 21 885-21 895, 1995.

McPherron, R. L. and Hsu, T.-S.: A comparison of substorms occurring during magnetic storms with those occurring during quiet times, J. Geophys. Res, 107(A9), 1259, doi:10.1029/2001JA002008, 2002.

Molinski, T. S.: Why utilities respect geomagnetically induced currents, J. Atmos. Sol.-Terr. Phys., 64, 1765-1778, 2002.

Press, W. H., Teukolsky, S. A., Vetterling, W. T., and Flannery, B P.: Numerical Recipes in C., The Art of Scientific Computing, Second Edition, Cambridge University Press, 2002.

Pulkkinen, A., Amm, O., Viljanen, A., and BEAR Working Group: Ionospheric equivalent current distributions determined with the method of spherical elementary current systems, J. Geophys. Res., 108(A2), 1053, doi:10.1029/2001JA005085, 2003a.

Pulkkinen, A., Thomson, A., Clarke, E., and McKay, A.: April 2000 storm: ionospheric drivers of large geomagnetically induced currents, Ann. Geophys., 21, 709-717, 2003b.

Pulkkinen, A., Lindahl, S., Viljanen, A., and Pirjola, R.: Geomagnetic storm of 29-31 October 2003: Geomagnetically induced currents and their relation to problems in the Swedish highvoltage power transmission system, Space Weather, S08C03, doi:10.1029/2004SW000123, 2005. 
Rosenqvist, L., Opgenoorth, H., Buchert, S., McCrea, I., Amm, O., and Lathuillere, C.: Extreme solar-terrestrial events of October 2003: High-latitude and Cluster observations of the large geomagnetic disturbances on 30 October, J. Geophys. Res., 110, doi:10.1029/2004JA010927, 2005.

Rostoker, G. and Phan, T. D.: Variation of auroral electrojet spatial location as a function of the level of magnetospheric activity, J. Geophys. Res., 91, 1716-1722, 1986.

Sornette, D.: Critical phenomena in natural sciences: chaos, fractals, selforganization, and disorder: concepts and tools, Springer, 2nd ed., 2004.

Tanskanen, E. I., Viljanen, A., Pulkkinen, T. I., Pirjola, R., Häkkinen, L., Pulkkinen, A., and Amm, O.: At substorm onset, $40 \%$ of AL comes from underground, J. Geophys. Res., 106, 13 119-13 134, 2001.

Tanskanen, E., Pulkkinen, T. I., Koskinen, H. E. J., and Slavin, J. A.: Substorm energy budget during low and high solar activity: 1997 and 1999 compared, J. Geophys. Res., 107, doi:10.1029/2001JA900153, 2002.
Tanskanen, E. I., Slavin, J. A., Tanskanen, A. J., Viljanen, A., Pulkkinen, T. I., Koskinen, H. E. J., Pulkkinen, A., and Eastwood, J.: Magnetospheric substorms are strongly modulated by interplanetary high-speed streams, Geophys. Res. Lett., 32, L16104, doi:10.1029/2005GL023318, 2005.

Viljanen, A., Amm, O., and Pirjola, R.: Modelling Geomagnetically Induced Currents During Different Ionospheric Situations, J. Geophys. Res., 104, 28 059-28 072, 1999.

Viljanen, A., Nevanlinna, H., Pajunpää, K., and Pulkkinen, A.: Time derivative of the horizontal geomagnetic field as an activity indicator, Ann. Geophys., 19, 1107-1118, 2001.

Viljanen, A., Pulkkinen, A., Amm, O., Pirjola, R., Korja, T., and BEAR Working Group: Fast computation of the geoelectric field using the method of elementary current systems and planar Earth models, Ann. Geophys., 22, 101-113, 2004.

Weigel, R. S., Klimas, A. J., and Vassiliadis, D.: Solar wind coupling to and predictability of ground magnetic fields and their time derivatives, J. Geophys. Res., 108(A7), 1298, doi:10.1029/2002JA009627, 2003.

Wintoft, P.: Study of the solar wind coupling to the time difference horizontal geomagnetic field, Ann. Geophys., 23, 1949-1957, 2005 . 